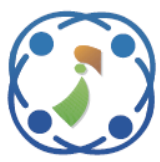

\title{
Multi-User Massive MIMO Systems Based on Hybrid Analog-Digital Beamforming for Millimeter-wave Communications
}

\author{
Mohammed K. Hussein ${ }^{1 *}$ \\ Nasser N. Khamiss ${ }^{2}$ \\ Information and Communication Engineering, Al-Nahrain University, Baghdad, Iraq \\ * Corresponding author's Email: mohammed.khudhur@gmail.com
}

\begin{abstract}
The rapid growth of traffic data will encounter future wireless communication. Unfortunately, the microwave spectrum cannot meet such rapid development. While Millimeter-wave (mm-wave) band can support the rapid growth of traffic owing to such band offers the enormous available bandwidth and multi-gigabit-per-second (Gbps) data rates. Therefore, a hybrid Beamforming solution (HBF) is a promising approach that exploits the small matrix digital precoding and the high matrix analog beamforming. This study addresses the question of how to effectively design and simulation of a low complexity hybrid precoding. It achieves near-fully digital precoding performance based on the hybrid minimum mean square error (MMSE) criterion and extraction of the angle phase for the analog beamforming as well as the Kalman precoding for the digital precoding. Thus, the results indicate that the proposed hybrid precoding has the best performance than the ZF hybrid precoding, the MMSE hybrid precoding, and the Kalman hybrid precoding in terms of computational complexity and spectral efficiency. The proposed solution achieves almost $2.257 \mathrm{bps} / \mathrm{Hz}$, at $20 \mathrm{~dB}$ with 3 number of paths and 3 number of scattering clusters compared to the Kalman precoding, almost $2.704 \mathrm{bps} / \mathrm{Hz}$ related to the MMSE precoding, and $2.95 \mathrm{bps} / \mathrm{Hz}$ compared to the Zero Forcing (ZF) under the same conditions. On the other hand, proposed precoding harvests $8.554 \mathrm{bps} / \mathrm{Hz}$ related to the analog beamforming while $-1.178 \mathrm{bps} / \mathrm{Hz}$ concerning the full digital precoding that is considered as an optimal value. This research has thrown up many questions in need of further investigation.
\end{abstract}

Keywords: Hybrid beamforming, Millimeter-wave, Multi-user massive MIMO, Kalman filter.

\section{Introduction}

Previous studies have found that the rapid growth of the traffic data will encounter the future wireless communication resulted in a 1000 -fold increase in capacity demand $[1,2]$. Unfortunately, the microwave spectrum cannot meet such rapid development, mainly high data rate, low end-to-end latency, and global mobile data traffic [3-5].

Recently, there has been renewed interest in the Millimeter-wave (mm-wave) communication range $28-300 \mathrm{GHz}$ for Fifth-generation (5G) wireless communication networks owing to such the band offers the enormous available bandwidth and multigigabit-per-second (Gbps) data rates. On the other hand, the $\mathrm{mm}$ - Wave band suffers from fundamental technical challenges owing to its short wavelengths. Fortunately, the mm-wave signals can encounter these challenges by combating a large number of antennas to be deployed in a small area. Herein, massive multiple-input multiple-output (massiveMIMO) plays a crucial role in enabling a large number of antennas [3, 6-9].

Throughput per user can be enhanced according to the key factors, which guide the performance of a cellular system. The fundamental equation that describes data throughput is as follows (Shannon eq.):

$$
R<C=m\left(\frac{B}{K}\right) \log _{2}(1+\mathrm{SINR})
$$

Where $B$ denotes the signal bandwidth, $k$ indicates the number of users who share the base station, $m$ indicates the spatial freedom between transmitter and receiver. SINR denotes the desired signal power to interference plus noise ratio at the receiver. 
The use of an extra spectrum as mm-wave extends $B$, which linearly increases the data capacity. Massive MIMO increases spatial links between transmitter and receiver by increasing the number of antennas at both sides. Cell splitting can reduce the $k$ factor by utilizing small cells and sectorization orders per site. Also, it increases both desired and interfering signals by getting the access point closer to the user $[10,11]$. To enable such growth, requiring a fundamental change in system architecture and radio technology by merging the $5 \mathrm{G}$ enablers (e.g., mmWave bands, massive MIMO, and ultra-dense SmallCells (SCs)) [12].

Based on large antenna arrays, the main challenge faced by many researchers is the fully digital beamforming (DBF) that is considered in sub- $6 \mathrm{GHz}$ will be unrealistic due to power and complexity issues. That is due to the dedicated radio frequency (RF) chain is equipped with a single antenna. On the other hand, analog beamforming (ABF) is an energyefficient solution. However, it is limited by one data stream that mainly leads to severe performance limitations in a multi-user scenario. Therefore, a hybrid Beamforming solution (HBF) is a promising approach that exploits the small matrix digital precoding and the high matrix analog beamforming to meet these challenges [13-16].

The remainder of the paper is organized as follows: Section 2 surveys related research articles, section 3 describes the system model, Section 4 analyses problem formulation, section 5 presents the proposed hybrid precoding scheme, and section 6 discusses the simulation results. Finally, the conclusion is reported in section 7 .

Notations: This paper considers $\mathrm{A}$ and $\mathbf{a}$ as a matrix and a vector. On the other hand, $\mathrm{A}^{\mathrm{H}}, \mathrm{A}^{-1}$, $\mathrm{A}^{\mathrm{T}},|\mathrm{A}|$, and $\|\mathrm{A}\|_{\mathrm{F}}$ represent Hermitian, inverse, transpose, determinant, and Frobenius norm of a matrix, respectively A. $\mathbb{C}$ is the field of complex numbers. Finally, $I$ and $\mathbb{E}[$.$] denote the identity$ matrix and the expectation operator.

\section{Literature survey}

The literature on multi-user mm - Wave MIMO systems shows a variety of approaches. In [17], authors propose two-stage hybrid precoding, in which the RF beamforming and combining are selected from predefined codebooks based on maximizing the array gain in the first stage. In the second stage, the zero-forcing (ZF) precoding is used to mitigate multi-user interference. The major drawback of this approach is complexity time because of the size of predefined codebooks. In a follow-up study, [18] separately selects the analog combining at each MS. After that, it uses an orthogonal matching pursuit to attain the RF precoding and then MMSE criterion to eliminate multi-user interference. A fundamental problem of this algorithm is in the practice applications. In [19], the authors propose the same method depended in [17] to attain the RF analog precoding and combining while applying iterative Kalman-based approach as the digital precoding to eliminate inter-user interference. As a result, the problem with this approach is also the size of codebooks. In [20] estimates analog and digital precoding via the general eigenvalue decomposition (GEVD) method. However, complexity is also a significant drawback. Another solution is described in [21], in which the hybrid precoding and combining are alternatively optimized. While the analog part is obtained via the GEVD, hence, this approach may not be practical. In [22], the authors suggest that RF precoding and combining exploit the large array gain. After that, the equivalent baseband channel is processed by Block diagonalization (BD) method to eliminate the interference. To maximize the data rate, [23] focuses on the effective channel to attain analog beamforming while the BD is applied to reduce the inter-user interference. In [24], the authors propose the Gram-Schmidt algorithm to obtain the analog beamforming. On the other side, a low dimensional effective channel is the basis for calculating digital precoding based on the MMSE method. These methods depend on orthogonalizing $\mathrm{ABF}$ vectors with the partial channel vectors. Thus, it is hard in practice due to phase shifts of accuracy is required.

Few published studies have explored the hybrid precoding solutions for the multi-users $\mathrm{mm}$ - Wave MIMO systems in the term spectral efficiency and computational efficiency. Besides, most of the previous works consider that full channel state information (CSI) is available on the transmitter. Nevertheless, in practical applications, only quantized CSI is considered through limited feedback.

In this paper, while we refer to earlier work, the focus is different. It proposes a new approach to design the hybrid precoder that overcomes the drawbacks of fully digital and analog beamforming. This research sheds new light on the integration of two methods to achieve the best result. Therefore, our approach includes some steps. Firstly, the proposed hybrid beamforming takes into account statistical CSI and applies the MMSE criterion to the aggregate downlink channel. After that, it extracts and treats with the angle phase to obtain the RF analog precoding and combining. Secondly, the Kalman precoding is applied to obtain digital precoding that is responsible for the cancellation of multi-user 
interference. Finally, under practical conditions, the proposed precoding investigates its performance based on the quantization bits.

The main contribution of our study are summaries as following:

1. Design and simulation of a low complexity hybrid precoding based on the MMSE criterion and extraction of the angle phase to obtain the RF analog precoding and combining. After that, the Kalman precoding is considered to attain digital precoding for the multi-users mm - Wave MIMO systems. Such a combination takes computational efficiency and spectral efficiency into account.

2. The results of this study indicate that the proposed solution outperforms some existing techniques (the ZF hybrid precoding [17], the MMSE hybrid precoding [18], and the Kalman hybrid precoding [19]) in terms of spectrum efficiency and computational efficiency under various conditions. Furthermore, one interesting finding is the suitable result of our algorithm under practical conditions with the quantization bits.

\section{System model}

On the download (DL), integration of the hybrid precoding solution and multi-users Massive MIMO technology based on the $\mathrm{mm}$ - Wave channel is given in Fig. 1. Such communication networks include the number of antenna NBS and NtRF RF chains at a base station (BS), in which each RF chain equipped with all antenna to serve $\mathrm{K}$ mobile stations (MSs) simultaneously to transmit Ns data streams. Each user has the number of antenna NMS and single RF chains to receive data stream. In order to simplify the problem, we assume that each user transmits singlestream transmission. Based on orthogonal multiple access, the number of RF chains must satisfy (K Ns $\leq \mathrm{NtRF} \leq \mathrm{NBS}$ and $\mathrm{Ns} \leq \mathrm{NrRF} \leq \mathrm{NMS}$ ) for the BS and the MSs.

At the BS, the digital beamforming precodes the signal vector followed by the analog beamforming that maps RF signal to $N_{B S}$ antennas. The transfer function between the BS and the user is written as:

$$
\begin{aligned}
& x_{k}=\underbrace{\sqrt{p_{k}} H_{k} F_{R F} F_{B B}^{K} S_{K}}_{\text {Desired signal }}+ \\
& \underbrace{\sum_{j \neq k}^{K} \sqrt{p_{k}} H_{k} F_{R F} F_{B B}^{j} S_{j}}_{\text {Interference signal }}+\underbrace{n_{k}}_{\text {Noise }}
\end{aligned}
$$

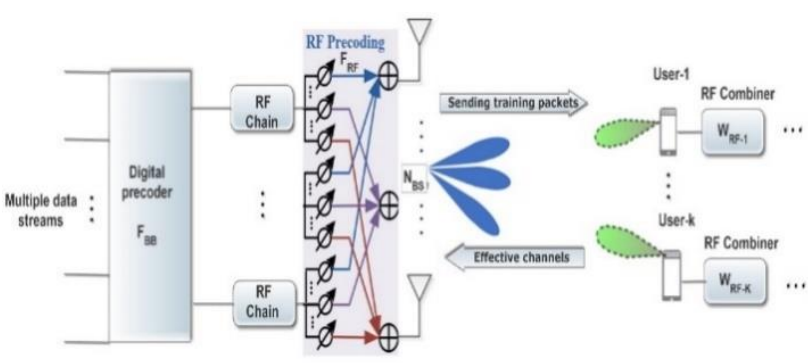

Figure. 1 Proposed hybrid precoding system

Where $p_{k}$ is transmission power, $H_{k} \in \mathbb{C}^{N_{M S} N_{B S} K}$ is the channel matrix between the $K$ users and the BS, $n_{k} \in \mathbb{C}^{K}$ is the noise vector, in which entries satisfy $\mathbb{E}\left[n n^{H}\right]=\sigma^{2} I_{K}, S_{k} \in \mathbb{C}^{K}$ is the signal vector, in which entries satisfy $\mathbb{E}\left[S S^{H}\right]=(p / k) I_{K}, F_{R F} \in \mathbb{C}^{N_{B S} K}$ is the analog precoding matrix, and $F_{B B} \in \mathbb{C}^{K}{ }^{K}$ is the digital precoding matrix for each user.

At the receiver, in order to obtain the signal RF and down-convert to the baseband signal, the received signals are combined through the matrix of the analog combiner. Thus, the received signal can be expressed as:

$$
\begin{aligned}
& y_{k}=\sqrt{p_{k}} H_{k} F_{R F} F_{B B}^{K} W_{K}^{H} S_{K}+ \\
& \sum_{j \neq k}^{K} \sqrt{p_{k}} H_{k} W_{K}^{H} F_{R F} F_{B B}^{j} S_{j}+W_{K}^{H} n_{k}
\end{aligned}
$$

Where $W \in \mathbb{C}^{N M S} K$ is the analog combiner. In terms of propagation nature, the $\mathrm{mm}$ - Wave channel is sparse that encounters a serious attenuation and severe multipath due to its small wavelength. A geometric channel model is to capture sparse scattering (Saleh-Valenzuela model) [25], as shown:

$$
\begin{gathered}
H=\sqrt{\frac{N_{B S} N_{M S}}{N_{c l} N_{r a y}}} \sum_{i=1}^{N_{c l}} \sum_{l=1}^{N_{r a y}} u_{i l} u_{M S} \\
\left(\varnothing_{i l}^{M S}, \theta_{i l}^{M S}\right) u_{B S}\left(\varnothing_{i l}^{B S}, \theta_{i l}^{B S}\right)
\end{gathered}
$$

Where $u_{M S}\left(\phi^{M S}, \theta^{M S}\right)$ and $u_{B S}\left(\phi^{B S}, \theta^{B S}\right)$ indicate array response vector for transmitting and receiving side whereas $\left(\phi^{B S}, \theta^{B S}\right)$ and $\left(\phi^{M S}, \theta^{M S}\right)$ represent azimuth and elevation angles of departure and arrival, respectively, and $u_{i l}$ is the complex gain of $N_{r a y}$ rays and $N_{c l}$ clusters for $K$ users. In our simulations, uniform plane array (UPA) and their array responses are given by:

$$
\begin{aligned}
& u_{B S}\left(\emptyset_{i l}^{B S}, \theta_{i l}^{B S}\right) \\
& =\frac{1}{\sqrt{N_{B S}}}\left[1, ., e^{-i \frac{2 \pi}{\lambda} d\left(\left(1-N_{B S-w}\right) \cos \left(\theta_{i l}^{B S}\right)\right)}\right. \\
& e^{\left.-i \frac{2 \pi}{\lambda} d\left(\left(1-N_{B S-h}\right) \sin \left(\emptyset_{i l}^{B S}\right) \sin \left(\theta_{i l}^{B S}\right)\right)\right]}
\end{aligned}
$$




$$
\begin{aligned}
& u_{M S}\left(\emptyset_{i l}^{M S}, \theta_{i l}^{M S}\right) \\
& =\frac{1}{\sqrt{N_{M S}}}\left[1, ., e^{-i \frac{2 \pi}{\lambda} d\left(\left(1-N_{M S-w}\right) \cos \left(\theta_{i l}^{M S}\right)\right)}\right. \\
& e^{\left.-i \frac{2 \pi}{\lambda} d\left(\left(1-N_{M S-h}\right) \sin \left(\varnothing_{i l}^{M S}\right) \sin \left(\theta_{i l}^{M S}\right)\right)\right]}
\end{aligned}
$$

Where $N_{h}$ and $N_{w}$ are numbers of UPA antennas indexes (height and width) for transmitter and receiver, $\lambda$ is the wavelength is the antenna spacing, which is usually scaled by wavelength $(\mathrm{d}=\lambda / 2)$.

The signal to interference plus noise ratio(SINR) of $\mathrm{K}$ user, is given as:

$$
R_{K}=\sum_{k=1}^{K} \log _{2}\left[1+\frac{\left|\sqrt{p_{k}} H_{k} F_{R F} F_{B B}^{K} W_{K}^{H}\right|^{2}}{\left|\sum_{j \neq k}^{K} \sqrt{p_{k}} H_{k} W_{K}^{H} F_{R F} F_{B B}^{j}\right|^{2}+\sigma^{2}}\right]
$$

\section{Problem formulation}

Our goal is the hybrid precoding and combining design that is optimization formulation. Besides, the BS digital precoder is based on the Kalman algorithm. It is to improve the average system rate. The sum-rate can be maximized, as expressed as follows:

$$
\begin{aligned}
& \underbrace{\max }_{F_{R F}, F_{B B}, W}=\sum_{k=1}^{K} \quad R_{K} \\
& \text { s.t. } \\
& \left\{\begin{aligned}
\left|\left(F_{R F}\right) i, j\right| & =\frac{1}{\sqrt{N_{B S}}} \\
\left|\left(W_{K}\right) i, j\right| & =\frac{1}{\sqrt{N_{M S}}} \\
\left\|F_{B B}\right\|_{F}^{2} & =K
\end{aligned}\right.
\end{aligned}
$$

The above nonconvex optimization problem is generally difficult to solve directly. Instead, the analog part (preceding and combining) design and digital component (precoding) design can be represented in two sub-problems.

Furthermore, the effective channel (antenna array gain) of the kth user is to tackle huge training signal overhead, as well it has a dimension smaller than the mm-wave channel matrix $H$ that can be written as:

$$
H_{e f f}=F_{R F} H_{k} W_{K}^{H}
$$

\section{Proposed hybrid precoding scheme}

In the first step, the MMSE criterion is applied to the aggregate downlink channel. The noise effect is considered by the MMSE that can achieve better performance compared with the Zero Forcing, as shown in the following equation:

$$
H_{x x}=\left(H_{K}^{H} H_{k}+\frac{K \sigma^{2}}{P} I\right) H_{K}^{H}
$$

Next, it is necessary to extract phases for the control on the alignment at the RF domain. Next, at the BS, the analog precoding $F_{R F}$ can be expressed as:

$$
F_{R F}^{K}=\frac{1}{\sqrt{N_{B S}}} e^{i(\operatorname{angle}(i, j))}
$$

Where angle $(i, j)$ is the unquantized phase of the $(i, j)$ th entity of the $H_{x x}$.

Based on the MMSE criterion also, the equations that describe the RF combiner is as follows:

$$
\begin{gathered}
A_{K}=F_{R F}^{K} H_{K} \\
H_{y y}=\left(A_{K}^{H} A_{K}+\frac{K \sigma^{2}}{P} I\right) A_{K}^{H}
\end{gathered}
$$

Then, the analog combining is described by Eq. (13) for all MS.

$$
W_{K}=\frac{1}{\sqrt{N_{M S}}} e^{i(\operatorname{angle}(i, j))}
$$

Where angle $(i, j)$ is the unquantized phase of the $(i, j)$ th entity of the $H_{y y}$.

Finally, the digital precoding is responsible for eliminating interference among users. However, it requires the existence of an effective channel. On the other hand, analog precoding and combining are calculated, as shown above. Now we can compute baseband precoding $\mathrm{F}_{B B}$ according to Kalman precoding with less iteration that can estimate unknown variables and then eliminate the multi-user interference. Thus, the error e(i) at iteration $i$ th can be computed as:

$$
E\{\operatorname{diag}(e(i))\}=\frac{I-H_{e f f} F_{B B}^{K}(i \mid i-1)}{\left\|I-H_{e f f} F_{B B}^{K}(i \mid i-1)\right\|^{2}}
$$

Next, baseband precoding $F_{B B}$ can be estimated and then normalized according to the relation, as shown:

$$
F_{B B}^{K}=\frac{F_{B B}^{K}}{\left\|F_{R F} F_{B B}^{K}\right\|_{F}}
$$

Under practical conditions, the proposed precoding scheme needs to investigate its performance based on the quantization bits that digitally control on phase shifters. However, this method results in system performance degradation. The RF precoding quantizes each phase of its elements up to $\mathrm{K}$ bits. 


\section{Simulation results}

In this simulation results section, our proposed algorithm is evaluated and compared with various techniques as the benchmark in terms of spectral efficiency and computational complexity. These techniques are analog beamforming, MMSE full digital precoding, the ZF hybrid precoding [17], the MMSE hybrid precoding [18], and the Kalman hybrid precoding [19]. For simulations, we used MATLAB R2019a. On the other hand, the results are more than 1000 random channel implementations on average.

Fig. 2 depicts the performance curves of the spectrum efficiency against the signal to noise ratio (SNR) with the number of BS antennas $N_{B S}=64$, the number of MS antennas $N_{M S}=4$, the number of users $\mathrm{K}=4$, the number of paths $=3$, and the number of scattering clusters $=3$. The analysis and simulation indicate that the fully digital system outperforms all techniques owing to an individual radio frequency (RF) chain is linked with a single antenna to serves multiple users at the same time. Nevertheless, hardware costs and energy consumption are the bottlenecks of that technique. On the other hand, only analog beamforming is not sufficient because one RF chain is used for all antennas. It is clear that the proposed algorithm outperforms the ZF hybrid precoding, the MMSE hybrid precoding, and the Kalman hybrid precoding. That is due to the Kalman approach being based on iterative precoding that adapts the baseband precoding matrix to eliminate multi-users interference. Besides, the MMSE criteria and the phase extraction technique that controls the angle phase for obtaining the analog beamforming instead of codebook with the large size.

Consider Fig. 3, which plots the curves of spectral efficiency versus the different number of users, in which $N_{B S}=64, N_{M S}=4, \mathrm{SNR}=10 \mathrm{~dB}$, the number of paths $=3$, and the number of scattering clusters $=3$. The performance of proposed precoding is better than different techniques, which are overloaded with the increase in the number of users. Nevertheless, the algorithms proposed gradually increase with the increase in the number of users. That is due to that the Kalman precoding with less iteration that can reduce inter-user interference underutilized the control on the phase compared with other algorithms. More details, an iterative Kalman based multi-user hybrid solution that minimizes the error between the preamble transmitted by the BS and the estimated received data at the MS. Furthermore, massive MIMO and hybrid precoding exploit large-scale antenna arrays at each base station (BS) and mobile stations (MSs). Therefore, it eliminates interference

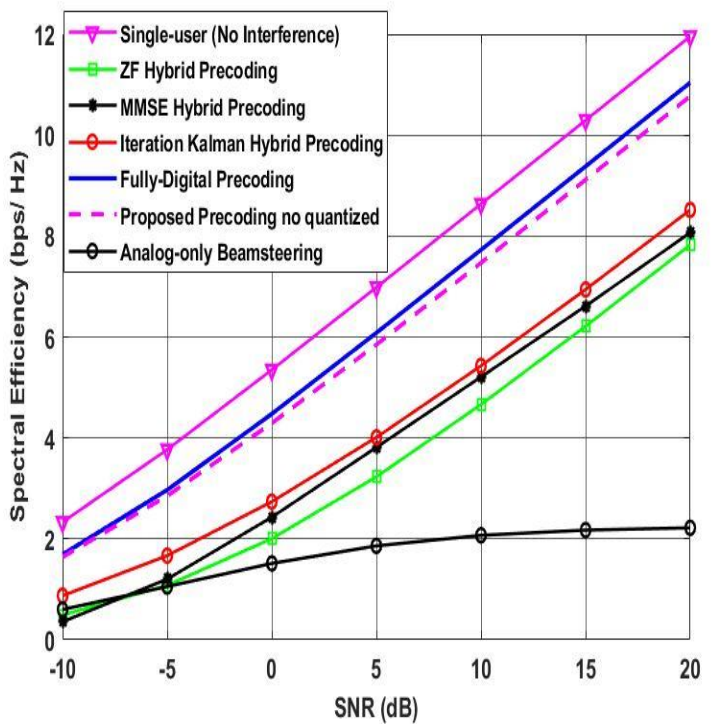

Figure. 2 The spectral efficiency versus the SNR

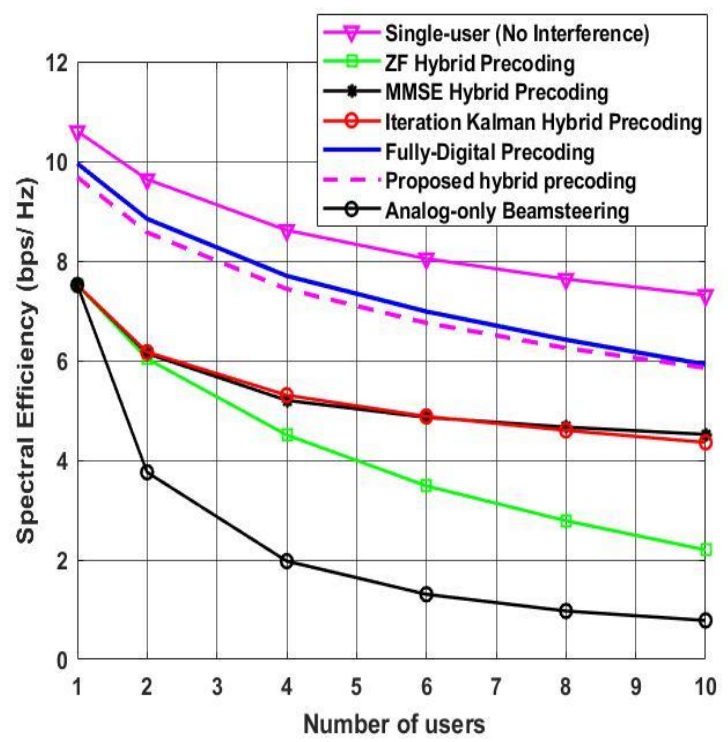

Figure. 3 The spectral efficiency versus the number of users

between users and achieves various benefits such as canceling out noise and fast fading through highly directional beamforming.

Fig. 4 shows spectral efficiency against the number of $B S$ antennas, in which, $S N R=10 \mathrm{~dB}, K=4$, the number of MS antennas NMS $=4$, the number of paths $=3$, and the number of scattering clusters $=3$. The increase in the number of BS antennas achieves a high data rate of all the approaches due to the massive MIMO, which employs a few hundred antennas at the BS, which is considered a reliable technique with many potential benefits compared with the analog combining at the MS. The number of antennas at BS is higher than in MS. However, the proposed algorithms harvest higher performance related to other techniques with the increase of the BS antennas. 


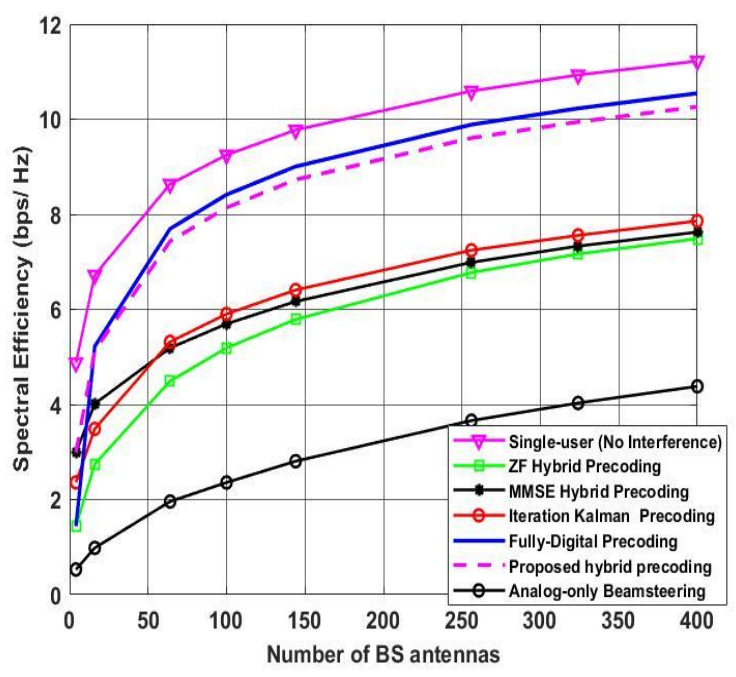

Figure. 4 The spectral efficiency versus the number of BS antennas

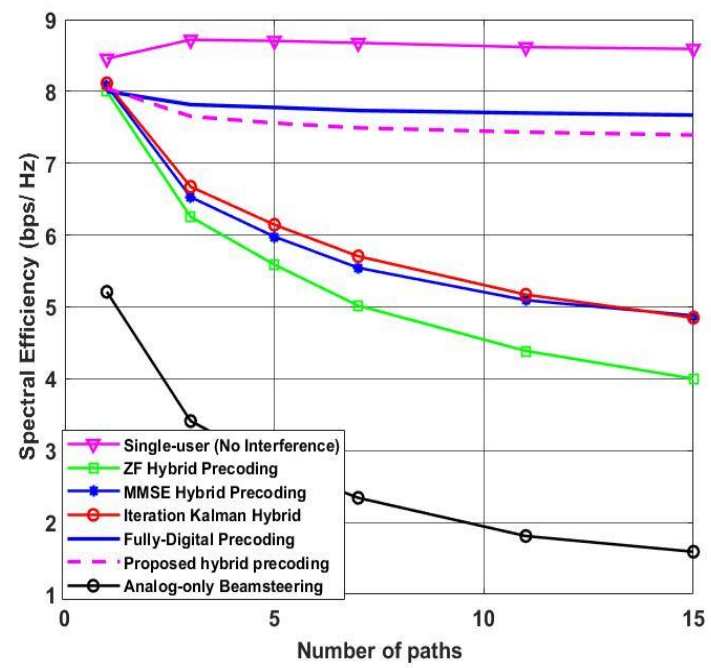

Figure. 5 The spectral efficiency versus the number of paths

For the resulting plot, see Fig. 5 shows spectral efficiency versus the number of paths, in which, $N_{B S}$ $=64, \mathrm{SNR}=10 \mathrm{~dB}, \mathrm{~K}=8$, and NMS $=4$. With the increase in the number of paths, it is a clear trend of slightly decreasing in the proposed algorithms compared with other algorithms that have significant performance degradation. That is mainly due to proposed algorithms more accurate beamforming than the other solutions. The results highlight that the proposed algorithms can effectively eliminate interuser interference.

Fig. 6 shows the performance curves of the spectrum efficiency against the SNR with $N_{B S}=64$, $N_{M S}=4, \mathrm{~K}=4$, the number of paths $=3$, the number of scattering clusters $=3$, and quantization bit $\mathrm{B}=1,2$. The result illustrates that even for coarse quantization, the proposed algorithm is still efficient; also, achieves

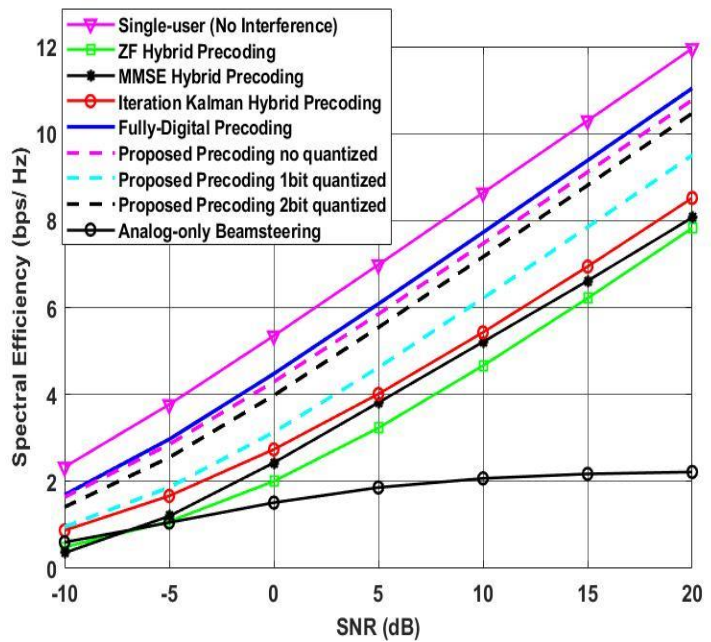

Figure. 6 The spectral efficiency versus the SNR

Table 1. Total complexity and spectral efficiency between different algorithms

\begin{tabular}{|l|c|c|}
\hline $\begin{array}{c}\text { Algorith } \\
\text { ms }\end{array}$ & Total complexity & $\begin{array}{c}\text { Spectral } \\
\text { efficiency }\end{array}$ \\
\hline $\begin{array}{l}\text { Zero } \\
\text { Forcing } \\
\text { HBF }\end{array}$ & $\sum_{k=1}^{K}\left(R^{2}+Z^{2}+K^{3}\right)$ & Low \\
\hline $\begin{array}{l}\text { MMSE } \\
\text { HBF }\end{array}$ & $\sum_{k=1}^{K}\left(R^{2}+Z^{2}+K^{3}\right)$ & Medium \\
\hline $\begin{array}{l}\text { Kalman } \\
\text { HBF }\end{array}$ & $\sum_{k=1}^{K}\left(R^{2}+Z^{2}+10 K^{3}\right)$ & Medium \\
\hline $\begin{array}{l}\text { Proposed } \\
\text { HBF }\end{array}$ & $\sum_{k=1}^{K}\left(R^{2}+K+R K^{3}\right.$ & High \\
\hline \multicolumn{2}{|l}{$\left.+5 K^{3}\right)$} & \\
Where $R=\max \left\{\mathrm{N}_{\mathrm{BS}}, \mathrm{N}_{\mathrm{MS}}\right\}$ and $Z^{2} \leq R$ \\
\hline
\end{tabular}

results are approximation close to no quantized algorithm.

Based on the computational analysis, we focus on the number of matrix multiplications to compare our proposed and other techniques. The results obtained from the preliminary analysis of different algorithms can be compared in Table 1 .

The results, as shown in Table 1, indicate that the proposed hybrid precoding has the best performance than other algorithms in terms of computational complexity and spectral efficiency. That is due to some existing solutions (the ZF hybrid precoding [17], the MMSE hybrid precoding [18], and the Kalman hybrid precoding [19]) depend on predefined codebooks that have the large size to compute the analog beamforming. That causes the complexity of 
time, while our solution takes a statistical method with a lower complexity for analog beamforming and the Kalman precoding with less iteration for the digital precoding. Thus, this a significant finding might be explained by the fact that the combination of the MMSE criteria and phase extraction technique along with the Kalman hybrid precoding can provide a reasonable method with low complexity.

As follows from the figures shown above, we examine proposed hybrid precoding under various conditions. It offers results that are in useful agreement with other studies.

Finally, merging $5 \mathrm{G}$ enablers that can overcome the rapid growth of the traffic data. On the other hand, the fundamental change will be considered in system architecture and radio technology. Also, the hybrid Beamforming solution is a promising approach that exploits the small matrix digital precoding and the high matrix analog Beamforming to meet these challenges.

\section{Conclusion}

The present study was undertaken to design hybrid precoding. It compares with other solutions in terms of computational complexity and spectral efficiency. Summing up the results, it can be concluded that the proposed hybrid precoding has the best performance than other algorithms. That is due to the Kalman approach based on iterative precoding that adapts the baseband precoding matrix to eliminate multi-users interference. Besides, the MMSE criteria and the phase extraction technique that controls the angle phase for obtaining the analog beamforming instead of codebook with the large size. Such a combination takes computational efficiency and spectral efficiency into account. Thus, our proposed algorithm achieves almost $2.257 \mathrm{bps} / \mathrm{Hz}$, at $20 \mathrm{~dB}$ with 3 number of paths and 3 number of scattering clusters compared to the Kalman precoding, almost $2.704 \mathrm{bps} / \mathrm{Hz}$ related to the MMSE precoding, and $2.95 \mathrm{bps} / \mathrm{Hz}$ compared to the Zero Forcing (ZF) under the same conditions. On the other hand, proposed precoding harvests $8.554 \mathrm{bps} / \mathrm{Hz}$ related to the analog beamforming while -1.178 $\mathrm{bps} / \mathrm{Hz}$ concerning the full digital precoding that is considered as an optimal value. These findings add to a growing body of literature on the hybrid precoding, mainly in terms of computational complexity and spectral efficiency. The proposed method can be readily used in practice. This research has thrown up many questions in need of further investigation. Based on the promising findings presented in this paper, work on the remaining issues is continuing and will be presented in future papers.

\section{Conflicts of Interest}

The authors declare that there is no conflict of interests regarding the publication of this paper.

\section{Author Contributions}

Conceptualization, M. K. H and N. N. K; methodology, M. K. H; software, M. K. H; validation, M. K. H and N. N. K; formal analysis, M. K. H; investigation, M. K. H and N. N. K; resources, M. K. $\mathrm{H}$; data curation, M. K. H; writing - original draft preparation, M. K. H; writing-review and editing, M. K. H and N. N. K; supervision, N. N. K.

\section{References}

[1] A. Hemadeh, K. Satyanarayana, M. El-Hajjar, and L.Hanzo, " MillimeterWave Communications: Physical Channel Models, Antenna Constructions, and Link-Budget ", IEEE Commun. Surv. Tutorials, Vol. 20, No. 2, pp. 870-913, 2018.

[2] R. Hasan, M. Mowla, A. Rashid, K. Hosain, and I. Ahmad, "A Statistical Analysis of Channel Modeling for 5G mmWave Communications", In: Proc. of Conf. Electr. Comput. Commun. Eng., pp. 1-6, 2019.

[3] A. Uwaechia and N. Mahyuddin, "Acomprehensive Survey on Millimeter-Wave Communications for Fifth-Generation Wireless Networks: Feasibility and Challenges", IEEE Access, Vol. 8, pp. 62367-62414, 2020.

[4] M. Shaheenbanu and M. N. Suma, "Investigatition on Hybrid Precoding for Multi-user MIMO Systems", In: Proc. of WIE Conf. Electr. Comput. Eng. WIECON-ECE - Proc., pp. 1-4, 2019.

[5] M. Hussein and N. Khamiss, "Integrating Millimeter-Wave with Hybrid Precoding MultiUser Massive MIMO for 5G Communication", Telecommunication Comput. Electron. Control., Vol. 18, No. 1, pp. 90-98, Feb.2020.

[6] H. M. Elmagzoub, "On the MMSE-Based Multiuser Millimeter Wave MIMO Hybrid Precoding Design", J. Commun. Syst., 2019.

[7] J. Du, J. Li, J. He, Y. Guan, and H. Lin, "LowComplexity Joint Channel Estimation for Multiuser Mm-Wave Massive MIMO Systems", Electron., Vol. 9, No. 2, pp. 1-18, 2020.

[8] J. Ortega, R. Sampaio-Neto, and R. David, "Novel Hybrid Precoder Based on SVD for Downlink Mmwave Massive MU-MIMO Systems", In: Proc. of 2019 IEEE Latin-American Conf. Commun. LATINCOM, pp. 1-28, 2019.

[9] J. Jin, C. Xiao, W. Chen, and S. Member, "Channel-Statistics-Based Hybrid Precoding for 
Millimeter-Wave MIMO Systems with Dynamic Subarrays", IEEE Trans. Commun., Vol. 67, No. 6, pp. 3991-4003, 2019.

[10] N. Al- and O. Alani, "Design considerations of ultra-dense $5 \mathrm{G}$ network in the millimeter-wave band", In: Proc. of Conf. Ubiquitous Futur. Networks, ICUFN, pp. 141-146, 2017.

[11] N. Bhushan, J. Li, and M. Malladi, "Network Densification: The Dominant Theme for Wireless Evolution into 5G", IEEE Commun. Mag., Vol. 52, No. 2, pp. 82-89, 2014.

[12] J. G. Andrews, S. Buzzi, and W. Choi, "What will 5G be?", IEEE J. Sel. Areas Commun., Vol. 32, No. 6, pp. 1065-1082, 2014.

[13] Z. Zhou, N. Ge, and Z. Wang, "HardwareEfficient Hybrid Precoding for Millimeter-Wave Systems with Multi-Feed Reflectarrays", IEEE Access, Vol. 6, No. c, pp. 6795-6806, 2018.

[14] M. Xiao, S. Mumtaz, and Y. Huang, "Millimeter -Wave Communications for Future Mobile Networks", IEEE J. Sel. Areas Commun., Vol. 35, No. 9, pp. 1909-1935, 2017.

[15] D. Carrera, S. Member, and C. Vargas-rosales, "Performance Improvement for Multi-User Millimeter-Wave Massive MIMO Systems", IEEE Access, Vol. 6, pp. 1-15, 2019.

[16] T. Lin, J. Cong, and Y. Zhu, "Hybrid Beamforming for Millimeter-Wave Systems Using the MMSE Criterion", IEEE Transactions on Communications, vol. 67, no. 5, pp. 3693-3708, 2019.

[17] A. Alkhateeb, G. Leus, and R. W. Heath, "Limited Feedback Hybrid Precoding for MultiUser Millimeter Wave Systems", IEEE Trans. Wirel. Commun., Vol. 14, No. 11, pp. 64816494, 2015.

[18] D. Nguyen, L. Le, and T. Le-Ngoc, "Hybrid MMSE Precoding for Mmwave Multi-User MIMO Systems", In: Proc. of IEEE International Conf. Erence on Comm. (ICC), pp. $1-6,2016$.

[19] A. Vizziello, P. Savazzi, and K. Chowdhury, "A Kalman Based Hybrid Precoding for Multi-User Millimeter Wave MIMO Systems", IEEE Access, Vol. 6, pp. 55712-55722, 2018.

[20] J. Cong, X. Li, and Y. Zhu, "Hybrid Precoding for Multi-User MmWave Systems Based on MMSE Criterion", In: Proc. Of Asia-Pacific Conference on Communications (APCC), 2017.

[21] J. Cong, T. Lin, and Y. Zhu, "Hybrid MMSE Beamforming for Multi-user Millimeter-Wave Communication Systems", IEEE Commun. Lett., Vol. 22, No. 11, pp. 2390-2393, 2018.

[22] W. Ni and X. Dong, "Hybrid Block Diagonalization for Massive Multiuser MIMO Systems",
IEEE Trans. Commun., Vol. 64, No. 1, pp. 201211, 2016.

[23] C. Hu, J. Liu, X. Liao, Y. Liu, and J. Wang, “A Novel Equivalent Baseband Channel of Hybrid Beamforming in Massive Multi-user MIMO Systems", IEEE Commun. Lett., Vol. 22, No. 4, pp. 764-767, 2018.

[24] J. Li, L. Xiao, X. Xu, and S. Zhou, "Robust and Low Complexity Hybrid Beamforming for Uplink Multiuser MmWave MIMO Systems", IEEE Commun. Lett., Vol. 20, No. 6, pp. 11401143, 2016.

[25] T.Rappaport, Millimeter-Wave Wireless Communications. Prentice-Hall, 2014. 